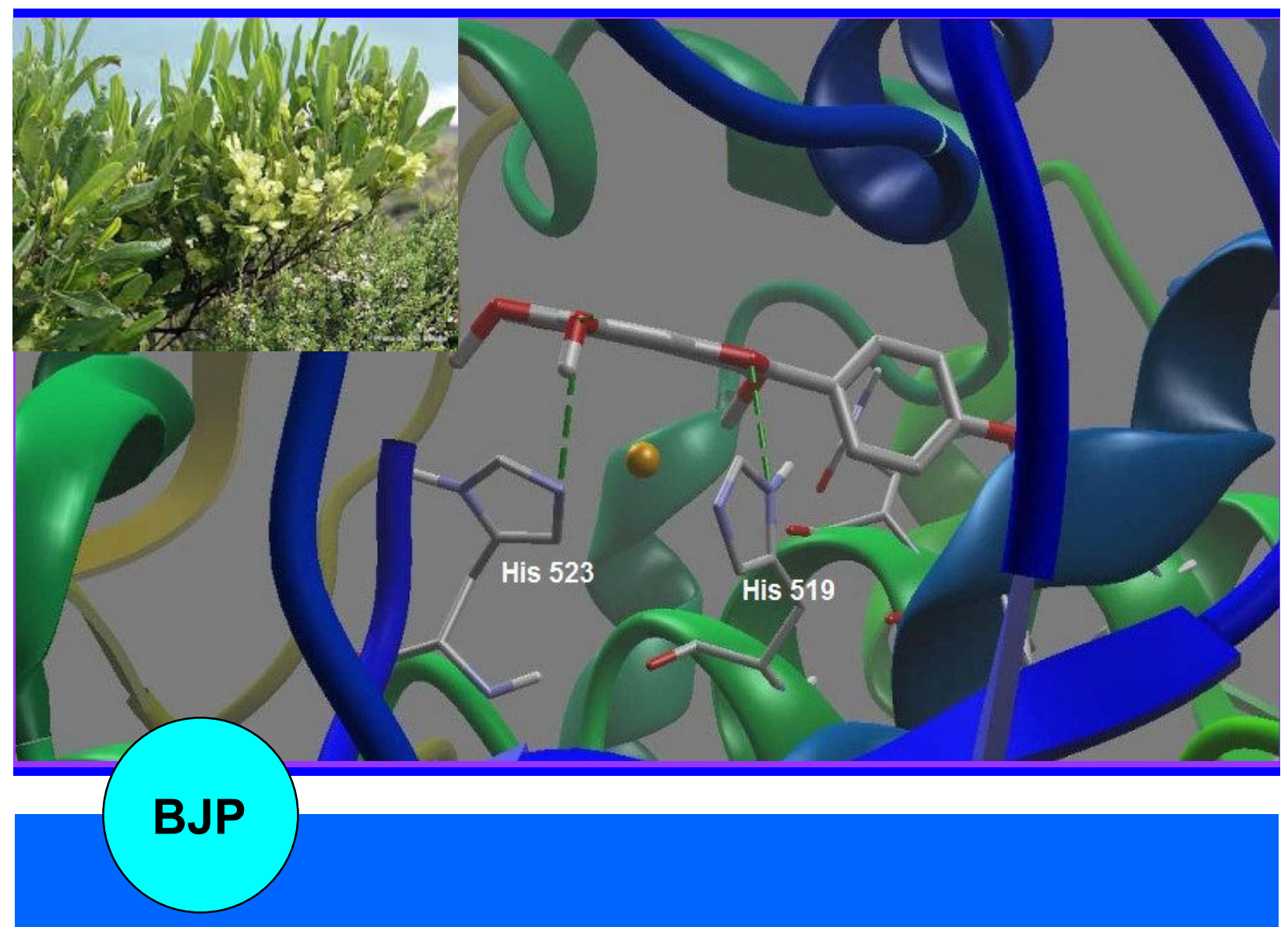

Bangladesh Journal of Pharmacology

Research Article

Molecular docking of viscosine as a new lipoxygenase inhibitor isolated from Dodonaea viscosa 


\section{Molecular docking of viscosine as a new lipoxygenase inhibitor isolated from Dodonaea viscosa}

\section{Amir Zada Khan', Akhtar Mohammad², Zafar Iqbal', Itrat Anis², Muhammad Raza Shah', Said Nadeem ${ }^{3}$, Muhammad Rabnawaz ${ }^{3}$, Adnan Shahidullah', Humayun Khan' and Inamullah Khan'}

${ }^{1}$ Department of Pharmacy, University of Peshawar, Peshawar 25120, Pakistan; ${ }^{2}$ Department of Chemistry, University of Karachi, Karachi 75270, Pakistan; ${ }^{3}$ H.E.J. Research Institute of Chemistry, International Center for Chemical and Biological Sciences, University of Karachi, Karachi 75270, Pakistan.

\begin{tabular}{|c|c|}
\hline \multicolumn{2}{|l|}{ Article Info } \\
\hline Received: & 23 December 2012 \\
\hline Accepted: & 2012 \\
\hline Available Online: & 31 December 2012 \\
\hline \multicolumn{2}{|c|}{ DOI: 10.3329/bjp.v8i1.13088 } \\
\hline \multicolumn{2}{|c|}{$\begin{array}{l}\text { Cite this article: } \\
\text { Khan AZ, Mohammad A, Iqbal Z, } \\
\text { Anis I, Shah MR, Nadeem S, Rab- } \\
\text { nawaz M, Shahidullah A, Khan H, } \\
\text { Khan I. Molecular docking of visco- } \\
\text { sine as a new lipoxygenase inhibitor } \\
\text { isolated from Dodonaea viscose. Bangla- } \\
\text { desh J Pharmacol. 2013; 8: 36-39. }\end{array}$} \\
\hline
\end{tabular}

\begin{abstract}
Viscosine was isolated from Dodonaea viscosa, which showed significant lipoxygenase inhibitory activity ( $\mathrm{IC}_{50}$ : value $\left.39 \pm 0.17\right)$. Molecular docking simulations were conducted to explore molecular binding mode, and to help elucidate molecular mechanism behind its significant inhibitory activity. Molecular interactions of viscosine with catalytic triad (His523, His518, Ile875) inside active site of lipoxygenase via hydrogen bonding, seems to be the major factor involved in its significant lipoxygenase inhibitory activity.
\end{abstract}

\section{Introduction}

Dodonaea, a genus of 60 species, is widely distributed in the warmer parts of most of the countries. Dodonaea viscosa (L.) Jacq. (syn-Ptelea viscosa Linn.), a flowering ever green shrub belongs to the family Spindaceae, which consists of about 150 genera and approximately 2,000 species. D. viscose is traditionally used as a folk medicinal preparations for various diseases and pathological conditions, which includes its use as an analgesic, anti-inflammatory, antiviral, spasmolytic, laxative, antimicrobial, hypotensive agent, rheumatism, gout, hemorrhoids, fractures and snake bites (Ghisalberti et al., 1998; Venkatesh et al., 2008). This plant is experimentally investigated for significant pharmacological activities of its crude extracts. Crude extracts of D. viscosa showed significant actions as local anesthetic, smooth muscle relaxant, antibacterial, anti-inflammatory, antifungal, anti-ulcerogenic, anti-ascariasis, ant- helmintic, cardiac depressant, hypotensive, uterine relaxation and vasoconstrictor activity in different experimental models (Venkatesh et al., 2008). In the current study a flavonoid (named viscosine) was isolated (Figure 1) with promising lipoxygenase inhibitory activity along with computational insights based on molecular docking. In the current study a flavonoid (named viscosine) was isolated (Figure 1) with promising lipoxygenase inhibitory activity along with computational insights based on molecular docking.

\section{Materials and Methods}

\section{Plant material}

The aerial parts of $D$. viscosa were collected from the hills of Kurram Agency, Khyber-Pakhtoonkhwa, Pakistan and identified by Dr. Ijaz Khan, a plant taxonomist at the Department of Botany, Post Graduate College, 


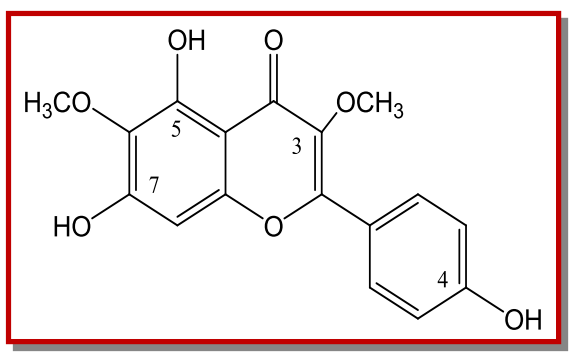

Figure 1: Chemical structure of viscosine

Kohat, Pakistan. A voucher specimen (DVPGCK-098) has been deposited in the herbarium of Department of Botany, Postgraduate College, Kohat, as reported earlier (Muhammad et al., 2012).

\section{Extraction and isolation}

The shade-dried plant material $(20 \mathrm{~kg})$ was ground into powder and extracted at room temperature with $\mathrm{MeOH}$ ( $35 \mathrm{~L} \times 3 \times 15$ days). After removal of the solvent, the extract was suspended in $\mathrm{H}_{2} \mathrm{O}$ and extracted with hexanes, $\mathrm{CHCl}_{3}, \mathrm{EtOAc}$, and $n-\mathrm{BuOH}$ to yield hexanes (116 g), $\mathrm{CHCl}_{3}(890 \mathrm{~g})$, EtOAc (173 g), and $n$-BuOH (337 g) extracts. The EtOAc extract was subjected to MPLC (medium pressure liquid chromatography) on silica gel and eluted with hexanes $/ \mathrm{CHCl}_{3}, \mathrm{CHCl}_{3} / \mathrm{EtOAc}$, EtOAc/ $\mathrm{MeOH}$, and $\mathrm{MeOH}$ to obtain 38 fractions (Fr. 1Fr. 38). The isolated compound (named viscosine, Figure 1) was confirmed by comparison with analytical data available in literature (van Heerden et al., 2000) $(3.8 \mathrm{~g})$ was purified from Fr. 23 (obtained with hexanes $\left./ \mathrm{CHCl}_{3}, 2: 8,5 \mathrm{~L}\right)$ by column chromatography [silica gel, hexanes/EtOAc $(6: 4,500 \mathrm{~mL})$ ].

\section{Lipoxygenase inhibition assay}

Enzyme inhibition assays were performed by using different concentrations of the isolated compound viscosine. Lipoxygenase inhibitory activity was measured by slightly modifying the spectrometric method as reported earlier (Khan et al., 2009; 2011). Lipoxygenase (EC 1.13.11.12) and linoleic acid were purchased from Sigma (St. Louis, MO) and were used without further purification. All other chemicals were of analytical grade and purchased from the same vendor i.e. Sigma (St. Louis, MO). $160 \mu \mathrm{L}$ of sodium phosphate buffer, $0.1 \mathrm{mM}$ ( $\mathrm{pH} 7.0), 10 \mathrm{~mL}$ of the sample solutions (test compound and standards) and $20 \mu \mathrm{L}$ of lipoxygenase solution were mixed and incubated for 5 min at $258^{\circ} \mathrm{C}$. Reaction was initiated by addition of 10 $\mu \mathrm{L}$ linoleic acid substrate solution and absorption change with formation of (9Z,11E)-13S)-13hydroperoxyo-ctadeca-9,11-dienoate was followed for $10 \mathrm{~min}$. Test sample and control were dissolved in 50\% ethanol. Baicalein and tenidap sodium were used as positive controls for lipoxygenase inhibition (Khan et al., 2009; 2011). The $\mathrm{IC}_{50}$ values were calculated as reported earlier (Khan et al., 2011). Viscosine showed significant inhibitory activity against lipoxygenase. $\mathrm{IC}_{50}$ values of viscosine, was $39.4 \pm 0.2 \mu \mathrm{M}$. Standard compound baicalein and tenidap sodium showed the $\mathrm{IC}_{50}$ value being $22.1 \pm 0.03 \mu \mathrm{M}$ and $41.6 \pm 0.02 \mu \mathrm{M}$.

\section{Molecular docking simulations}

Ligand file (viscosine) was designed and optimized using dreading force field was implemented in Marvin Sketch V5.1. Molecular coordinates were further optimized using MMFF force field. FRED 2.1 (Khan et al., 2011) was used to dock the OMEGA pre-generated multi-conformer library. De-fault FRED protocol was used except for the size of the box defining the binding sites. In an attempt to optimize the docking-scoring performance, exhaustive docking was performed with shapegauss applying the "Optimization" mode. The "Optimization" mode involves a systematic solid body optimization of the top ranked poses from the exhaustive docking. 3 different boxes were explored for lipoxygenase (PDB ID: 1JNQ). Three different simulations were carried out with an added value of 9 $\AA$ around the active site. After completion, best scoring pose was selected to study molecular interactions behind significant enzyme inhibitory activity of viscosine.

\section{Results and Discussion}

Lipoxygenase (EC 1.13.11.12) constitutes a family of non-heme iron containing enzymes, as versatile biocatalysts are capable of catalyzing many reactions involved in xenobiotic metabolism. They are responsible for the metabolism of the fatty acids (FAs) and their metabolites eliciting inflammatory responses in the body. They also play a significant role in cancer cell growth, metastasis, invasiveness, cell survival and induction of tumor necrosis factor (TNF) (Khan et al., 2009; 2011). Catalytic or active site of lipoxygenase is composed of 3 important amino acid residues the socalled catalytic triad (His523, His518, Ile875) along with iron atom (Khan et al., 2011). Any compound, which is capable to show strong molecular interaction with the catalytic triad, can be developed as new and therapeutically effective lipoxygenase inhibitor. According to results of Molecular docking analysis, viscosine showed slightly better binding energy in comparison to baicalein. The best scoring poses of viscosine, baicalein, and tenidap were -76.1, -93.3, -69.8, Kcal/mol, respectively.

According to predicted binding affinities of all compounds were comparable with the experimental inhibitory effects of compounds. In order to explore molecular interactions of viscosine with lipoxygenase, results of docking simulations were studied. Viscosine showed strong molecular interactions with all important amino acid residues inside active site of lipoxygenase especially the catalytic triad (Figure 2 and 


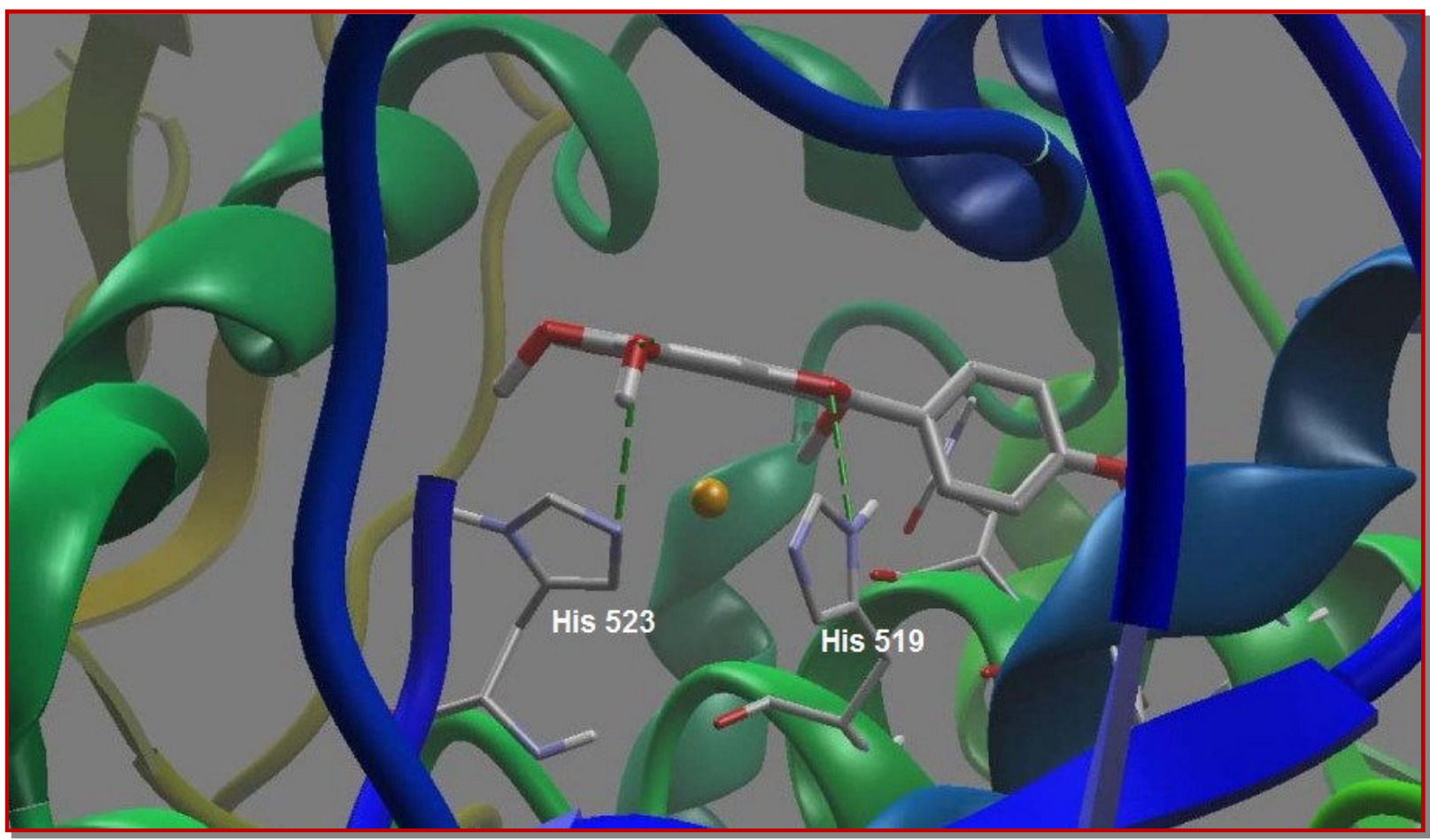

Figure 2: Binding mode of viscosine inside catalytic site of lipoxygenase. Orange colored round object is Fe atom. Hydrogen bonding (green dotted lines) are selectively (only for His523 and His519, for details see Figure 3) shown for clarity. Hydrogen atoms (except polar ones) were omitted for clarity

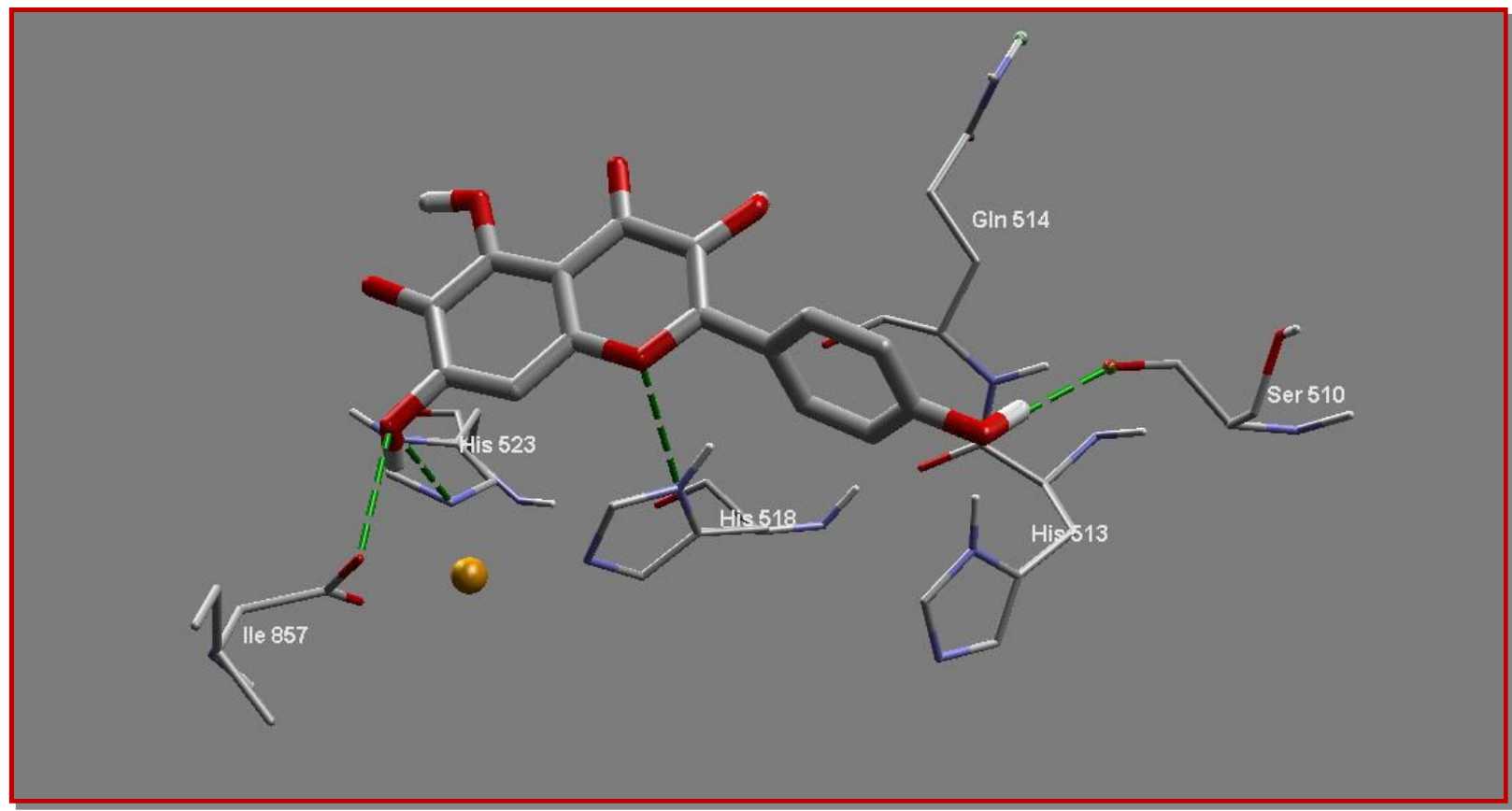

Figure 3: Closer view of molecular interactions of viscosine inside catalytic site of lipoxygenase. Orange colored round object is Fe atom. Hydrogen atoms (except polar ones) were omitted for clarity

Figure 3). Phenolic group at C-7 of the bicyclic aromatic core of viscosine seems to be the most important group of viscosine indicating its simultaneous and dual hydro -gen bonding interactions with Ile857 and His523 at a distance of distance of $2.77 \AA$ and $3.24 \AA$, respectively. Double-sided hydrogen bonding interaction of $\mathrm{OH}$ group at C-7, rendered the bicyclic aromatic core to vertically suspend over the space between catalytic 
triad and iron (Fe) atom. In the same binding mode, phenolic group also exhibited favorable dipole-cation interactions (Figure 2). The combined hydrogen bonding and dipole-cation interactions appear to be the most important interactions involved in potent inhibitory activity of viscosine as a lipoxygenase inhibitor. Heterocyclic oxygen atom showed hydrogen bonding with His518 at a distance of $2.91 \AA$. The terminal phenolic moiety (i.e. 4-hydroxyphenyl group) of viscosine was identified to provide a firm and favorable support to ligand-protein complex via hydrogen bonding with His513, at a distance of $3.07 \AA$. Interestingly, the same phenolic group was also found to be in favorable contact with Ser510 via hydrogen bonding (at a distance of $3.20 \AA$ ). Apart from hydrogen bonding, dipole-dipole attraction was also found between phenolic moiety and amino group of Ser510 of the same position (4hydroxyphenyl group) at a distance of $3.4 \AA$. The whole scenario shows the critical role of terminal phenolic group with perpendicular confirmation (with respect to chromenone or bicyclic aromatic nucleus) for viscosine to be an active lipoxygenase inhibitor. Owing to the presence of positively charged Fe atom and surrounding amino acid residues bearing positive electrostatic interactions (Figure 3) favored the negatively charged electrostatic molecular surface of viscosine. Steric interactions between both the ligand and protein were supported by molecular shape of the viscosine snatching the binding pocket (active site) of lipoxygenase. Aside from hydrogen bonding, dipole-dipole, chargedipole, steric and electrostatic interactions, unfavorable hydrophobic-hydrophilic interactions were not found, which supported the molecular mechanism based on binding mode of viscosine on lipoxygenase. This significant inhibitory effect of viscosine could be a starting point of discovery of new and more effective derivatives of viscosine as lipoxygenase inhibitors. The test compound has considerable profile as a potential lead compound for treatment and management of inflammation and related pathological conditions associated with leukotrienes.

\section{References}

Ghisalberti EL. Ethnopharmacology and phytochemistry of Dodonaea species. Fitoterapia 1998; 69: 99-113.

Khan I, Nisar M, Shah MR, Shah H, Gilani SN, Gul F, Abdullah SM, Ismail M, Khan N, Kaleem WA, Qayum M, Khan HO. Anti-inflammatory activities of Taxusabietane A isolated from Taxus wallichiana Zucc. Fitoterapea 2011; 82: 1003-07.

Khan I, Nisar M, Ebad F, Nadeem S, Saeed M, Khan H. Antiinflammatory activities of Sieboldogenin from Smilax china Linn: Experimental and computational studies. J Ethnopharmacol. 2009; 121: 175-77.

Muhammad A, Anis I, Ali Z, Awadelkarim S, Khan A, Khalid A, Shah MR, Galal A, Khan IA, Choudhary MI. Methylenebissantin: A rare methylene-bridged bisflavonoid from Dodonaea viscosa which inhibits Plasmodium falciparum enoyl-ACP reductase. Bioorganic Med Chem Lett. 2012; 22: 610-12.

Muhammad A, Anis I, Khan A, Marasini BP, Choudhary MI, Shah MR. Biologically Active C-Alkylated Flavonoids from Dodonaea viscosa. Archiv Pharmacol Res. 2012; 35: 431-36.

Utar Z, Majid MIA., Adenan MI., Jamil MFA, Lan TM. Mitragynine inhibits the COX-2 mRNA expression and prostaglandin E2 production induced by lipopolysaccharide in RAW264.7 macrophage cells. J Ethnopharmacol. 2011; 136: 75-82.

van Heerden FR, Viljoen AM, van Wyk BE. The major flavonoid of Dodonaea angustifolia. Fitoterapia 2000; 71: 60204 .

Venkatesh S, Reddy YSR, Ramesh M, Swamy MM, Mahadevan $\mathrm{N}$, Suresh B. Pharmacognostical studies on Dodonaea viscosa Leaves. Afr J Pharm Pharmacol. 2008; 2: 83-88.

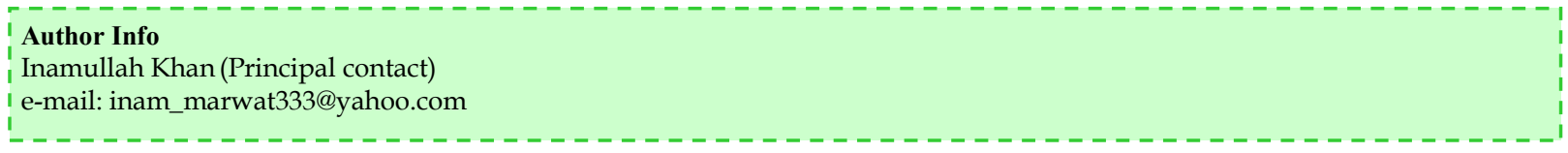

